\title{
EVALUATION OF SHEAR BOND STRENGTH OF HIGH-PERFORMANCE POLYMERS TO ITS RESIN VENEERING AND TO DENTIN (IN VITRO STUDY)
}

Mahinour A. Yousry ${ }^{1} B D S$ Sanaa A. Hussein ${ }^{2} P h D$, Fayza H. Al Abbassy ${ }^{3} P h D$

\begin{abstract} structure remains difficult. composite using two different adhesives, as well as to dentin using two types of cements. hardening adhesive). Subgroup IB: using Combo.lign (dual-hardening adhesive). of failure. KEYWORDS: Polyetheretherketone, PEEK, Shear Strength, Composite Resins.
1. B.D.S 2009
2. Professor of fixed prosthdontic, Faculty of Dentistry, Alexandria University
3. Assistant Professor of Dental Biomaterials, Faculty of Dentistry, Alexandria University

INTRODUCTION: : Looking through the wide range of prosthetic options High-performance polymers properties. Needs over layering resin veneer, bonded by a special adhesive, this adds an additional challenge to achieve adequate bond strength, also its bonding to tooth

OBJECTIVES: To evaluate shear bond strength of CAD/CAM High-performance polymers (BioHPP) with its CAD/CAM veneering

MATERIALS AND METHODS: This in vitro study, for Group I, twenty BioHPP discs with their CAD/CAM veneering composite, were milled and divided randomly into two subgroups $(\mathrm{n}=10)$ according to the adhesive system used. Subgroup IA: using DTK adhesive (dual-

For Group II, twenty CAD/CAM High-performance polymers (BioHPP) discs were milled and divided into two subgroups according to the different cements used to lute to dentin surface. Subgroup IIA: using: RelyX Unicem resin cement, subgroup IIB: using Fuji Plus GI cement. After water storage, thermocycling $\left(1200\right.$ cycles, $\left.5^{\circ} \mathrm{C} / 55^{\circ} \mathrm{C}\right)$ corresponding to one year of clinical service, all the specimens were subjected to shear force until failure and the results in the various groups were compared and statistically analyzed. Modes of failure were assessed. RESULTS: Mean SBS were as follows: subgroup IA (DTK adhesive 6.238), subgroup IB (combo.lign2.42), subgroup IIA (RelyX Unicem 2.07) and subgroup IIB (Fuji Plus 3.77). Mann-Whitney test demonstrated significant differences between the two subgroups in groups I and II $(\mathrm{U}=2.0, \mathrm{P}=.001)(\mathrm{U}=17, \mathrm{P}=.013)$ respectively. Stereomicroscope revealed that mixed and adhesive failure were the most dominant mode

CONCLUSIONS MDP-Containing Self-adhesive Resin (DTK adhesive) is recommended as efficient adhesive to increase the shear bond strength of the veneering composite to BioHPP. Also Fuji Plus showed better bond strength than RelyX Unicem with BioHPP and dentin.

\section{INTRODUCTION}

The goal of every efficient practice is to provide the most durable restorations possible, while exceeding the minimum aesthetic needs of the patient. A wide range of innovative and reliable materials is now available to satisfy the needs of both patients and dentists.

The original material, Polyetheretherketone (PEEK) is a semi-crystalline high-performance plastic combines very good mechanical properties, as high temperature resistance and excellent resistance to chemicals; it has attracted attention in medicine and is now gaining ground in the dental field. In medicine, PEEK has long been used in orthopedics and trauma management and for spinal cord implants. It remains stable when exposed to most organic and inorganic chemical agents. It also has several applications in dentistry as implant, temporary abutment or implant-supported bar or clamp due to its excellent physical and biological properties, it is used in fixed prosthodontics as well (1).

PEEK is off-white and without fillers or additives, it offers many advantages: it is a high purity material which has elastic properties similar to those of human bone; it is absolutely non-corrosive, radiolucent and has a low water absorption rate even after more than ten years of implant application, also due to its shock absorbing properties, this high-performance material is well suited for use in implant technology.
High Performance Polymer (BioHPP) is a PEEK variant that is high-temperature semi crystalline thermoplastic polymer from the main group of polyacryletherketone (PAEK), consisting of an aromatic backbone molecular chain, interconnected by ketone and ether functional groups with the density of 1.3-1.5 $\mathrm{g} / \mathrm{cm} 3(2)$. Thanks to its strengthening with special ceramic filler, optimized mechanical properties have been created for its use in fabrication of fixed prostheses. This ceramic filler has a grain size of 0.3 to $0.5 \mu \mathrm{m}(3)$.

The High Performance Polymer (BioHPP) framework can be fabricated using thermo pressing procedures or milled with CAD/CAM technique. Industrially fabricated CAD/CAM blocks have better mechanical and optical properties than conventional thermo pressed frameworks.

However, there are some aesthetic drawbacks that limit the use of PEEK as full-coverage monolithic restorations. The optical properties of PEEK included low translucency and a greyish color. Therefore a veneering layer, using additional resin composites is required. Veneering composite can be built directly by layering technique on the core after application of adhesive and light cured or it can be milled by CAD/CAM technique, and boned to the core by a special adhesive.

Adequate bond strength between veneering resin composite and BioHPP surface may be a challenge, due to 
its low surface energy and resistance to surface modification by different chemical treatments.

Also a recent study observed no bond between untreated BioHPP surfaces and resin luting cement (4). The manufacturer recommended pretreating the surface either by chemical method using sulfuric acid or mechanical treatment using air abrasion with aluminum oxide of grain size $110 \mu \mathrm{m}$, this increased the initial bond strength significantly.

This study was planned to evaluate the shear bond strength of CAD/CAM High-performance polymers with its $\mathrm{CAD} / \mathrm{CAM}$ veneering composite using two different adhesives, and to assess its shear bond to dentin using two different types of cement.

\section{MATERIALS AND METHODS}

In lab Forty CAD/CAM BioHPP (Bredent, Senden, Germany) discs were designed through the Zirkonzahn.Software (Modellier, Zirkonzahn, Italy) and became ready to be Connected with the Roland DWX-50 milling machine(Roland DG Corporation, Japan) for milling following the manufacturer's instructions and divided into two main groups $(n=20)$, Group I were used for evaluation of shear bond strength of CAD/CAM Highperformance polymers (BioHPP) core discs $(10 \mathrm{~mm}$ diameter, $4 \mathrm{~mm}$ thick) with its $\mathrm{CAD} / \mathrm{CAM}$ veneering composite discs (BreCAM.HIPC, Bredent, Senden, Germany) (4 mm diameter, $6 \mathrm{~mm}$ height) using two different types of adhesives: DTK adhesive (Bredent, Senden, Germany) and combo.lign (Bredent, Senden, Germany) and Group II were used for evaluation of the shear bond strength of BioHPP core discs (4 mm diameter, $6 \mathrm{~mm}$ height) with dentin surface using two different types of cements : dual-cure resin cement RelyX Unicem Aplicap (3M ESPE, RelyX Unicem Aplicap, St. Paul, Minn,USA) and Resin reinforced Glass Ionomer GC Fuji Plus (GC America Inc.).

\section{Specimens’ preparation for Group I}

Twenty (BioHPP) Core disc specimens(10 mm diameter, 4 $\mathrm{mm}$ thick) were milled and embedded in an autopolymerizing acrylic resin mold, using a rectangular split copper mold constructed with six circular holes, each one with $15 \mathrm{~mm}$ internal diameter and $2 \mathrm{~cm}$ depth. The rectangular split copper mold was assembled by interlocking between male and female parts. These holes were filled with self-cure polymethylmethacrylate resin and the discs were fixed in the resin while it was in the dough stage to be together in one plain. The surface of the discs together with the acrylic blocks were finished and smoothed by a wet 400-grit silicon carbide paper (Matador,Wasserfest,Germany), to be flushed together and to be absolute flat.

The exposed BioHPP surface in acrylic resin molds was sandblasted with aluminium oxide $(110 \mu \mathrm{m})$ at 2 to 3 bars blasting pressure (2) according to manufacturers' instructions, then Visio-link Primer (Bredent, Senden, Germany) was applied on the surface of samples using a microbrush and light cured for 90 seconds in bre.lux Power Unit(Bredent, Senden, Germany).

Twenty HIPC veneer material discs $(4 \mathrm{~mm}$ diameter, $6 \mathrm{~mm}$ height) were milled and made absolutely flat using the same technique. The twenty CAD/CAM BioHPP core disc specimens were then randomly divided into two sub groups $(n=10)$ according to type of adhesive material used to bond BioHPP discs with its veneering composite cylinders :

Subgroup IA: Core and veneer discs were bonded with DTK dual-hardening adhesive composite

Subgroup IB: Core and veneer discs were bonded with Combo.lign dual-hardening adhesive composite.

Cementation was done following manufacturers recommendations.

The HIPC veneer discs was placed over BioHPP disc and pressed together under a 5 kilogram fixed load and kept in place for 2 minutes to assure complete setting of the cement. Using a static load applicator (Custom made in fixed prosthodontic Department, Faculty of Dentistry, Alexandria University), the load was applied to keep the specimen in place until setting of the resin (figure.1). Excess cement was removed using a scaler.

\section{Specimens’ preparation for Group II Teeth Surface Preparation}

Twenty premolar teeth extracted for orthodontic treatment were collected. Teeth were only included in the study if they were free of dental caries and restorations. After extraction, the teeth were washed under running water, manually cleaned with periodontal curettes, disinfected using chloramine-B-hydrate for 1 week, and stored for two weeks in distilled water till used.

Each extracted premolar was then cut horizontally (perpendicular to the long axis of the tooth) using a diamond cutting disc under copious water cooling to produce a flat dentin surface in the occlusal surface and the roots were cut. A rectangular split copper mold was constructed with six circular holes, each one with $15 \mathrm{~mm}$ internal diameter and $5 \mathrm{~cm}$ depth. The hole was filled with self-cure polymethylmethacrylate resin (Acrostone Dental \& Medical Supplies, Egypt). While the acrylic resin was in the dough stage, the prepared tooth was embedded into it and centralized into the acrylic resin exposing the occlusal dentin surface only until polymerization occurred. The cut surfaces of the teeth together with the acrylic blocks were finished and smoothed by wet 400-grit silicon carbide paper so as to be flushed together, creating an absolute flat surface.

The twenty milled (BioHPP) Core discs specimens (4 $\mathrm{mm}$ diameter, $6 \mathrm{~mm}$ height) were air abraded according to manufacturers' instructions with aluminium oxide particles $(110 \mu \mathrm{m})$ at 2 to 3 bars blasting pressure for 5 seconds (2).

The fabricated twenty CAD/CAM BioHPP discs were randomly divided into two sub groups $(n=10)$ according to type of cement material used to lute BioHPP discs with dentin:

Group II was divided into two sub groups $(\mathrm{n}=10)$ Subgroup IIA: Core discs were luted with dentin surface using dual-cure resin cement (RelyX Unicem).

Subgroup IIB: Core discs were luted with dentin surface using Resin reinforced Glass Ionomer (Fuji plus).

Cementation was done following manufacturers recommendations.

The (BioHPP) Core discs were bonded to the tooth surface and pressed together under a 5 kilogram fixed load and kept in place for 4 minutes to assure complete setting of the cement. Using a static load applicator Figure 1, the load was applied to keep the specimen in place until setting 
of the resin. Excess cement was removed at the first formation of gel stage using a scaler.

\section{Aging of the specimens}

Thermocycling was done in a custom made thermocycling machine (Custom made in Dental Biomaterials Department, Faculty of Dentistry, Alexandria University). All specimens were cycled for 1200 thermal cycles in hot and cold water baths between $5-55^{\circ} \mathrm{C}$ in 1 minute cycle for each bath, with a dwell time of 30 seconds corresponding to one year of clinical service (5).

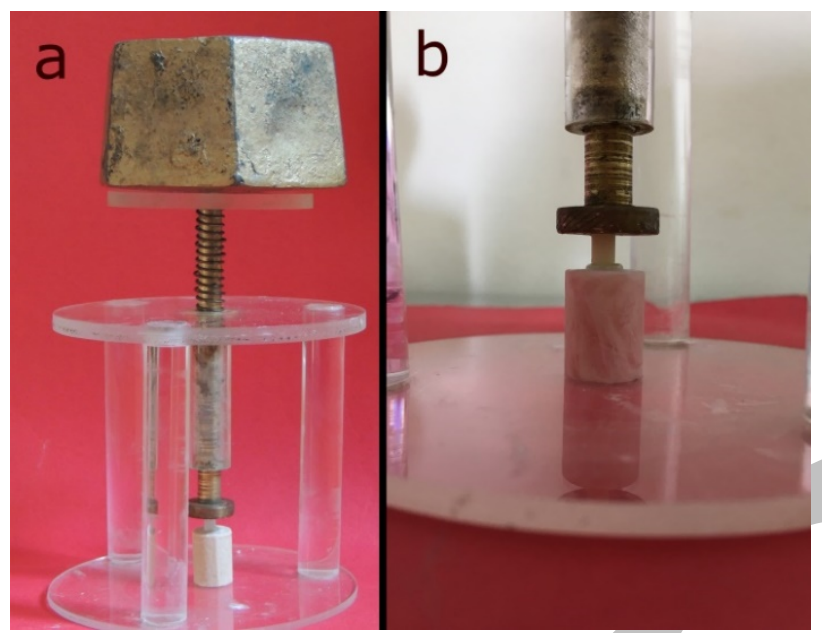

Figure (1): a: Bonding specimen under static load applicator, b: Close-up of bonded specimen.

\section{Shear Bond Strength Test - SBS}

The shear bond strength testing was done using a Universal Testing Machine (Comten industries, Fodida, USA). Each specimen was tightened in a metal mold in the lower platform of the universal testing machine. The specimens were oriented so that the stainless steel blade of the universal testing machine was perpendicular to the junction between the BioHPP disc and HIPC veneer disc for Group I and between tooth specimen and BioHPP disc for Group II. The blade was used to apply load at a cross head speed of $0.5 \mathrm{~mm} / \mathrm{min}$ until de-bonding. The load at which the debonding occurred was recorded for each specimen in both groups (Figure 2,3).
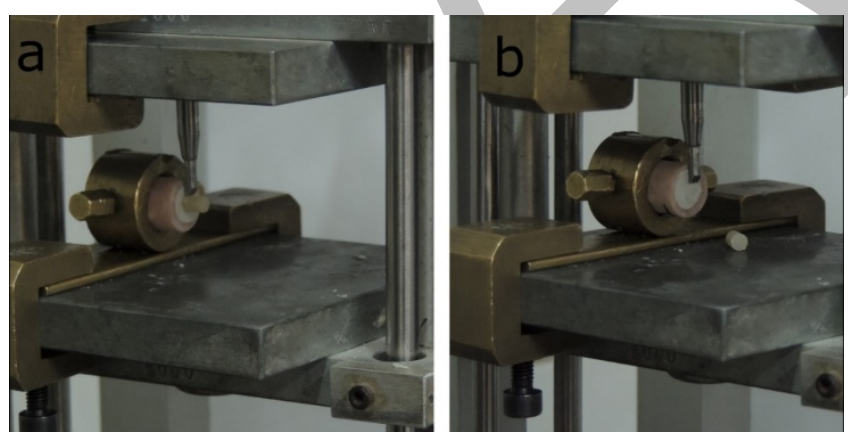

Figure (2): A specimen aligned with metal knife of Universal Testing Machine a) during load application, b) after debonding.

The Shear bond strength was calculated according to the following equation (6):

Shear stress $(\mathrm{MPa})=$ Load $(\mathrm{N}) /(\pi \times \mathrm{r} 2) \mathrm{N} / \mathrm{mm} 2$

Where, л $=3.14$

$\mathrm{r}=$ radius of the specimen in $\mathrm{mm} 2$.

$\mathrm{N}=$ load in Newton.

Failure Mode Analysis
After the test, all specimens of the two groups were carefully inspected at the debonded surfaces using the stereomicroscope to evaluate the pattern of bond failure. Stereo-microscope was connected to digital camera which captured images of the deboned surfaces at a magnification of 1.8X. For group I, Failure modes were classified into four types (7): (1) Adhesive failure mode between PEEK and resin materials, (2) Cohesive failure mode within PEEK, (3) Cohesive failure mode within resin materials, and (4) Mixed failure mode with both cohesive and adhesive failures.

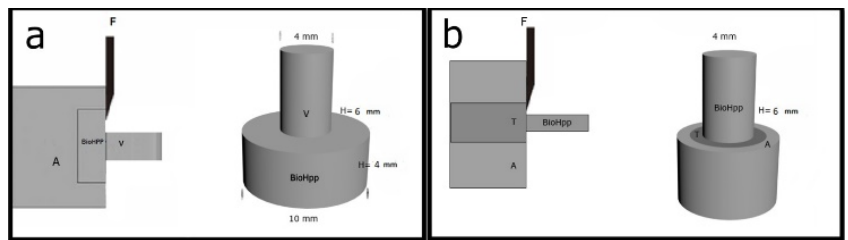

Figure (3): A diagram illustrating the shear bond strength test setup and specimen sizes, where BioHPP: High-performance polymers, V: veneering composite, A: acrylic special mold, F: force, T: tooth. a) For Group I, b) for Group II.

For group II, The failure mode was defined as follows (8): adhesive cement/dentin (no resin cement remnants left on the dentin surface), adhesive cement/PEEK (no resin cement remnants left on the PEEK surface), cohesive in dentin (the fracture occurred in the dentin), cohesive in PEEK (fracture occurred in the PEEK), mixed (resin cement remnants partially left on the PEEK and dentin surface with PEEK and dentin surface exposed).

\section{Statistical analysis}

The data collected from each group was tabulated descriptive analysis of each group. The mean value and standard deviation of each group was statistically analyzed using Statistical Package for Social Sciences [SPSS Version 20] software. Mann-Whitney test was used to reveal if there were significant differences between groups.

\section{RESULTS}

The means of shear bond strength in MPa for both Groups and the descriptive statistical analysis are shown in tables $(1,2)$.

Group I: The higher mean bond strength was recorded for subgroup IA (DTK adhesive) (6.238 $\pm 2.1 \mathrm{MPa})$, while the subgroup IB (Combo.lign) showed a mean value 2.42 \pm $0.79 \mathrm{MPa}$.

Mann-Whitney test revealed a significant difference between the two subgroups; $(\mathrm{U}=2.0, \mathrm{P}=.001$ ). Subgroup IA showed significant higher shear bond strength value compared to subgroup IB. P and U value are shown in table (1).

Group II: The means of shear bond strength in MPa for both groups and the descriptive statistical analysis are shown in table (2). The higher mean shear bond strength value was recorded in subgroup IIB (Fuji Plus) (3.77 \pm 1.63 $\mathrm{MPa}$ ), while the lower mean was recorded in subgroup IIA (RelyX Unicem) (2.07 $\pm 0.95 \mathrm{MPa})$.

Mann-Whitney test revealed a significant difference between the two subgroups; ( $\mathrm{U}=17, \mathrm{P}=.013)$. Subgroup IIB showed significant higher shear bond strength value compared to subgroup IIA. P and U value are shown in table (3). 


\section{Mode of Failure}

Visual examination of the fractured surfaces of Group I, Group II showed that mixed and adhesive fractures between PEEK and resin materials / dentin were the common types of failure for all the subgroups.

Adhesive failure type I observed in the subgroups as the following (Figure 5):

Table (1): Means of Shear bond strength in (MPa) of subgroups IA, IB in Group I.

\begin{tabular}{|c|c|c|c|c|}
\hline & $\begin{array}{c}\text { subgroup IA } \\
(\mathbf{n}=\mathbf{1 0})\end{array}$ & $\begin{array}{c}\text { Subgroup IB } \\
(\mathbf{n}=\mathbf{1 0})\end{array}$ & $\mathbf{U}$ & $\mathbf{P}_{\mathbf{1}}$ \\
\hline $\begin{array}{l}\text { Shear bond } \\
\text { strength (MPa) } \\
\text { Min. - Max. }\end{array}$ & $3.18-9.25$ & $1.19-3.41$ & & \\
Mean \pm SD. & $6.24 \pm 2.29$ & $2.42 \pm 0.79$ & $2.0^{*}$ & $<0.00$ \\
Median & 5.71 & 2.56 & $1^{*}$ \\
\hline
\end{tabular}

P1 : $p$ value for Mann Whitney test for comparing between subgroup IA and IB

$*$ : Statistically significant at $\mathrm{p} \leq 0.001$

Table (2): Means of shear bond strength in (MPa) for subgroups IIA, IIB in Group II.

\begin{tabular}{|c|c|c|c|c||}
\hline & $\begin{array}{c}\text { Subgroup } \\
\text { IIA } \\
(\mathbf{n = 1 0 )}\end{array}$ & $\begin{array}{c}\text { Subgroup } \\
\text { IIB } \\
(\mathbf{n}=\mathbf{1 0})\end{array}$ & $\mathbf{U}$ & $\mathbf{P}_{\mathbf{2}}$ \\
\hline $\begin{array}{c}\text { Shear bond } \\
\text { strength (MPa) } \\
\text { Min. - Max. }\end{array}$ & $1.06-3.46$ & $1.42-5.66$ & & \\
Mean \pm SD. & $2.07 \pm 0.95$ & $3.77 \pm 1.64$ & 17. & $<0.0$ \\
Median & 1.75 & 4.12 & $0^{*}$ & $13^{*}$ \\
\hline
\end{tabular}

$\mathrm{P}_{2}$ : $\mathrm{p}$ value for Mann Whitney test for comparing between subgroup IIA and IIB.

*: Statistically significant at $\mathrm{p} \leq 0.013$.

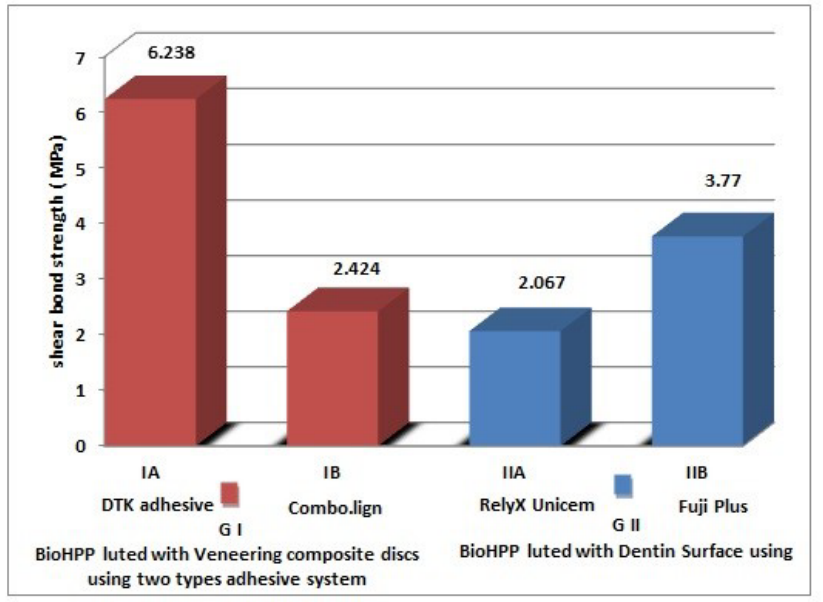

Figure (4): Histogram showing the mean shear bond strength in $\mathrm{MPa}$ for different subgroups in Group I and Group II.

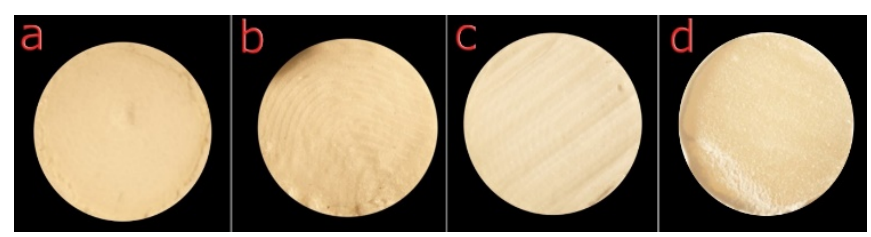

Figure (5): a) Stereomicroscope images of adhesive failure mode I in 4 specimens of Group I and Group II. a) in DTK adhesive subgroup IA, b) in combo.lign subgroup IB, c) in RelyX subgroup IIA, d) in Fuji Plus subgroup IIB.
Subgroup IA using DTK adhesive 5 specimens (50\%), subgroup IB using Combo.lign had 5 specimens (50\%), subgroup IIA RelyX 6 specimens (60\%) and subgroup IIB Fuji Plus 4 specimens $40 \%$.

Mixed failure observed in the subgroups as the following (Figure 6):

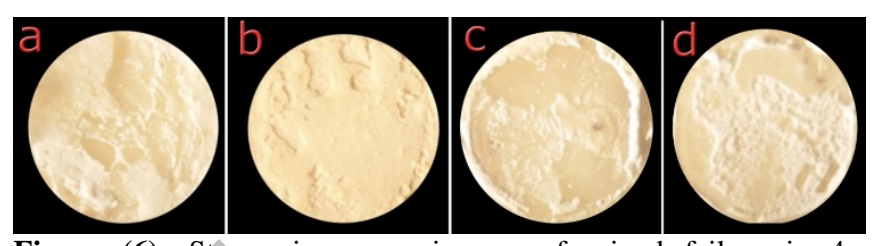

Figure (6): Stereomicroscope images of mixed failure in 4 specimens of Group I and Group II.a) DTK adhesive subgroup IA, b) Combo.lign subgroup IB, c) in RelyX subgroup IIA, d) in Fuji Plus subgroup IIB.

In subgroup IA within 5 specimens (50\%), subgroup IB within 6 specimens $60 \%$, subgroup IIA 4 specimens (40\%) and 5 specimens (50\%) in subgroup IIB.

There was one specimen type IV mode of failure (pure cohesive failure at the dentin) in subgroup IIB Fuji Plus tested specimen.

\section{DISCUSSION}

Polyetheretherketone (PEEK) is a material that has attracted attention in medicine and is now gaining ground in the dental field. Studies have focused on promoting the nonreactive PEEK surface to react with resins since PEEK is an apolar and inert polymer with high chemical resistance and low surface energy. Therefore, enhancing the surface properties of PEEK is an important priority, which needs to be addressed.

All tested PEEK discs in this study were sandblasted with $110 \mu \mathrm{m} \mathrm{Al} 2 \mathrm{O} 3$ at pressure of 2 to 3 bars as recommended by Stawarczyk et al. (4), who reported that PEEK specimens air abraded with $110 \mu \mathrm{m}$ alumina yielded higher roughness in comparison to the specimens abraded with $50 \mu \mathrm{m}$ alumina.

PEEK and composite veneer were fabricated using CAD/CAM technology since it was stated by Stawarczyk et al. (14) that industrial pre-pressing process for the CAD/CAM blanks increased the stability of PEEK and its veneering restorations and reduced risk of porosities and therefore showed improved mechanical properties. In contrast, the mechanical properties of pressed restorations are more operator-dependent: the preheating method, the vacuum pressing device and other factors may influence the overall quality of the specimens.

In this study regarding group I, surface roughness was shown to play an important role for adhesive technique combined with using a bonding agent. Nevertheless, roughening of the surface alone with blasting is not sufficient to guarantee a stable bonding between the composite and the PEEK surface this was in accordance to Stawarczyk et al. (15) and Hallmann et al (16).

Priming agent (visio.link) is a MMA-based adhesive system; it can be assumed that MMA caused the PEEK surface to swell and that the dimethacrylate monomers provided the connection to the composite resins with 2 carboxyl groups as binding sites. Most of the recent publications demonstrated that an adequate chemical bond to PEEK could be established with it (17-21). The contents and solvents of the adhesive system play important roles in 
creating a bond to PEEK. Three previous studies examined the bond strength properties of PEEK materials and found that adhesive systems that contain MMA monomers, such as visio.link or Signum PEEK Bond, resulted in the higher bond strengths (22-25).

The DTK adhesive luting agents promotes higher bonding to CAD/CAM High-performance polymers (BioHPP) with its CAD/CAM veneering composite with pretreatment of the surface by airborne-particle abrasion and the application of adhesive systems such as visio.link when compared with Combo.lign with shear bond strength SBS $(6.238 \pm 2.1 \mathrm{MPa})$ which exceeded the 5-MPa minimum clinically acceptable SBS value for bonded polymer restorations set by the International Organization for Standardization. Also DTK adhesive was statistically significant when compared with Combo.lign (26). This depicted that DTK adhesive which is classified as a resin based dual cure adhesive composite, the methacrylate monomers in it are partially substituted by phosphate monomers, such as MDP, which can promote chemical adhesion between the adhesive and the substrate. This appeared to be a determining factor in the stability and durability of bonds (27-30).

MDP has bifunctional ends that consist of long organic hydrophobic chain molecules, hydrophilic phosphate ester groups at one end bond strongly to pentaerythritol triacrylate (PETIA) of the primer, and vinyl groups react with the monomers of the resin at the other end, that is why DTK adhesive bonding technique might have some advantages in bonding. DTK adhesive has a flexual strength of 80 MPA and an adhesive strength of $350 \mathrm{~N}$ according to DIN EN ISO 15223-1 (30).

The result was in agreement with another study conducted by Stawarczyk et al. (31), who showed that applying the adhesive/composite system, the bond strength value of Sandblasting group with a mean grit size of $50 \mu \mathrm{m}$ was $8.7 \pm 2.8 \mathrm{MPa}$, similar results for bond strengths were obtained after air abrasion by Martin Rosentritt et al. (32), who concluded that good bonding between PEEK and composite, cleaning and roughening is recommended. Nine systems provided SBS higher than $5 \mathrm{MPa}, \mathrm{Al} 203-50 \mu \mathrm{m}$ groups were varying from $2.5 \pm 2.1$ to $8.8 \pm 2.7 \mathrm{MPa}$.

Regarding group II, RelyX unicem resin cement was selected for this study to see if the phosphoric acid group in the self-adhesive cement formulation was able to condition the surface of PEEK. Also because it is generally accepted that dual cure resin cements produce lower polymerization shrinkage stresses and therefore provide good marginal integrity and low micro-leakage as mentioned by Rosentritt et al. (33). Furthermore, Albert and El-Mowafy (34) explained that the insoluble resin cement absorbs water which may help the relaxation of the internal stress caused by polymerization shrinkage, consequently this reduces the potential of internal failure of the resin cement during thermo-cycling (35).

Under the conditions of this study, the results showed that it was not possible to achieve a strong bond to PEEK using phosphate containing monomer (RelyX Unicem) or resin-modified glass-ionomer (GC Fuji Plus) cements, but Fuji Plus has been showed better shear bond strength than RelyX Unicem.

The bond strength of Fuji Plus (3.77 $\pm 1.63 \mathrm{MPa})$ has

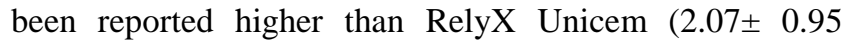
$\mathrm{MPa})$. However, the results of this study showed lower shear bond strength values (mean value $<10 \mathrm{MPa}$ in the two studied groups). All the bonding strength values in this study were below minimum clinically acceptable (36) SBS value 10-13 MPa set by the International Organization for Standardization (37).

In agreement to the current study, Patrick R. et al (2014) (38) evaluated the bond strength of differently pretreated and conditioned PEEK crowns luted to dental abutments. The results showed shear bond strength means (SBS) between PEEK and dentin in the airborne-particle abrasion groups were as follows: Signum PEEK Bond group $2.97 \pm 0.92 \mathrm{MPa}$; Visio. Link group was $2.12 \pm 0.78$ MPa; Ambarino P60 group and Without bond group 2.09 $\pm 0.58 \mathrm{MPa}$.

Additionally the latter result was in agreement with another study conducted also by Regina Furbino et al. (8) who showed mean shear bond strength (SBS) values with sandblasting was $2.37 \pm 0.86 \mathrm{MPa}$.

This was in disagreement with Schmidlin et al.(2) who obtained higher results for shear bond strengths when using the adhesive system Heliobond and RelyX Unicem, the result was $13.5 \pm 2.4 \mathrm{MPa}$.

The exact mechanism of adhesion of this material is not completely established. Some SEM (stereo electron microscope) studies revealed the formation of tags at the dentin-cement interface resultant from the Resin Reinforced Glass Ionomer (RMGIC) polymer penetration into the dentinal tubules (39). The application of surfacealtering solutions to dentin prior to bonding with glassionomer cements has been reported by many authors to increase the bond strength (40). Both Resin Reinforced Glass Ionomer and self-adhesive resin cements set by an acid-base reaction as well as a free radical polymerization reaction (39).

Fuji Plus Conditioner is a mild polyacrylic acid solution designed to remove the dentinal smear layer and to condition dentin and enamel before the application. It will increase the bond between glass ionomer cement and tooth structure for added longevity. These can explain the higher result of Fuji Plus cementing agent than RelyX Unicem.

Storage and thermo-cycling are the most often used artificial aging methods for simulating fatigue in laboratory testing of bonding durability. This minimal requirement of simulating oral conditions seems necessary before clinical recommendations can be provided. So before testing the specimens, thermal cycling regime was conducted to simulate inta-oral temperature changes on the test specimens during service for 1200 cycles corresponding to one year of clinical sevice, at $550 \mathrm{C}$ and $50 \mathrm{C}$. according to Rosentritt et al. (38).

Regarding mode of failure in the current study, the results revealed that, the specimens displayed mixed and adhesive failure were the most dominant mode of failure in the two groups.

Concerning to group I, the adhesive mode of failure resin material/veneer (no resin cement remnants left on the veneer surface), further proved the hypothesis that the reason for the observed pure adhesive breakdown lies in the pre-treatment. The airborne-particle abrasion increases the surface area and allows a better infiltration of the adhesive material and the application of an adhesive system (conditioning with visio.link) to allow for chemical interactions with BioHPP and the resin material, therefore 
creates a stronger bonding in all cases investigated in this study. This result was in accordance with Patrick et al. (40), who observed Mixed (adhesive/cohesive) failure types were found more frequently with in all the tested specimens of the airborne-particle abrasion group in combination with visio.link.

In group II, the adhesive mode of failure seems that the cement/dentin interface was stronger than the cement/PEEK interface in such groups. This may be due to the fact that the shear strength of the resin cement and the bond strength of the resin cement to the tooth dentin are higher than the results obtained for the bond strength of the resin cement to BioHPP.

\section{CONCLUSION}

Within the limitations of the present study, the following conclusions can be drawn:

1. MDP-Containing Self-adhesive Resin (DTK adhesive) promotes durable bonding to CAD/CAM Highperformance polymers (BioHPP) with its CAD/CAM veneering composite with pretreatment of the surface by airborne-particle abrasion and the application of adhesive systems such as visio.link.

2. Resin Reinforced Glass Ionomer (Fuji Plus) showed better shear bond strength than Dual-cure Resin Cement (RelyX Unicem Aplicap) with CAD/CAM Highperformance polymers (BioHPP).

\section{CONFLICT OF INTEREST}

The authors declare that they have no conflicts of interest.

\section{REFERENCES}

1. Kurtz SM, Devine JN. PEEK biomaterials in trauma, orthopedic, and spinal implants. Biomaterials. 2007;28:4845-69.

2. Schmidlin PR, Stawarczyk B, Wieland M, Attin T, Hämmerle $\mathrm{CH}$, Fischer $\mathrm{J}$ et al. Effect of different surface pretreatment and luting materials on shear bond strength to PEEK. Dent Mater. 2010; 26:553-9.

3. Jurgen V, Marc S, Frank S. BioHPP - a metal-free material for prosthetic restorations. Zahntech MAG. 2013;17:138-43.

4. Stawarczyk B, Beuer F, Wimmer T, Jahn D, Sener B, Roos M, et al. Polyetheretherketone- a suitable material for fixed dental prostheses. J Biomed Mater. 2013;101:1209-16.

5. Rosentritt M, Behra M, Zel J, Feilzer A. Approach for evaluating the influence of laboratory simulation. Dent Mater. 2009;25:348-52.

6. International Organization for Standardization. Dental materials -Testing of adhesion, ISO 11405, 2003.

7. Patcharawan S, Pisaisit Ch, Kittipong T, Banjong Ch, Takahashi H, Arksornnukit M. Effect of surface pretreatments on resin composite bonding to PEEK. Dent Mater J. 2016;35:668-74.

8. Regina F, Villefort R, Lilian C, Tiago M, Bastos C, Renata $\mathrm{M}$, et al. Bonding of the Polymer Polyetheretherketone (PEEK) to human dentin: effect of surface treatments. Braz Dent J. 2016;27:693-9.

9. Ourahmoune R, Salvia M, Mathia TG, Mesrati N. Surface morphology and wettability of sandblasted PEEK and its composites. Scanning. 2014;36:64-75.
10.Sproesser O, Schmidlin PR, Uhrenbacher J, Roos M, Gernet W, Stawarczyk B, et al. Effect of sulfuric acid etching of polyetheretherketone on the shear bond strength to resin cements. J Adhes Dent. 2014;16:46572.

11.Stawarczyk B , Bahr $\mathrm{N}$, Beuer $\mathrm{F}$, Wimmer $\mathrm{T}$, Eichberger M, Schmidlin P, et al. Influence of plasma pretreatment on shear bond strength of self-adhesive resin cements to polyetheretherketone. Clin Oral Invest. 2014;18:163-70.

12. Hossein P, Maryam R, Dastjerdi S, Vahid S, Alaleh S, Atefeh R, et al. Effect of Different Surface Treatment on Shear Bond Strength of Veneering Composite to Polyetherketone Core Material. Inter J of Adv Biotec \& Research. 2016;7:1116-21.

13.Kern M, Lehmann F. Influence of surface conditioning on bonding to Polyetheretherketone (PEEK). Dent Mater. 2012;28:1280-3.

14. Stawarczyk B, Eichberger M, Uhrenbacher J, Wimmer T, Edelhoff D, Schmidlin PR, et al. Three-unit reinforced polyetheretherketone composite FDPs: influence of fabrication method on load-bearing capacity and failure types. Dent Mater J. 2015;34:7-12.

15. Stawarczyk B, Bahr N, Beuer F, Wimmer T, Eichberger $\mathrm{M}$, Schmidlin PR, et al. Influence of plasma on shear bond strength of self-adhesive resin cements to polyetheretherketone. Clin Oral Investig. 2014;18:16370 .

16. Hallmann L, Mehl A, Sereno N, Hämmerle C. The improvement of adhesive properties of PEEK through different pretreatments. Applied Surface Science 2012;258:7213-8.

17. Heimer S, Schmidlin PR, Stawarczyk B. Discoloration of PMMA, composite, and PEEK. Clin.Oral Investig. 2016;21:1191-200.

18. Uhrenbacher J, Schmidlin PR, Keul C, Eichberger M, Roos M, Gernet W, et al. The effect of surface modification on the retention strength of polyetheretherketone crowns adhesively bonded to dentin abutments. J Prosthet Dent. 2014;112:1489-97.

19, Stawarczyk B, Bahr N, Beuer F, Wimmer T, Eichberger M, Gernet W, et al. Influence of plasma pretreatment on shear bond strength of self-adhesive resin cements to polyetheretherketone. Clin Oral Investig. 2014;18:16370 .

20.Kern M, Lehmann F. Influence of surface conditioning on bonding to Polyetheretherketone (PEEK). Dent Mater. 2012;28:1280-3.

21.Liebermann A, Wimmer T, Schmidlin PR, Scherer $H$, Loffler P, Stawarczyk B, et al. Physicomechanical characterization of polyetheretherketone and current esthetic dental CAD/CAM polymers after aging in different storage media. J. Prosthet Dent. 2016;115: 321-8.

22. Stawarczyk B, Keul C, Beuer F, Roos M, Schmidlin PR. Tensile bond strength of veneering resins to PEEK: impact of different adhesives. Dent Mater J. 2013;32:441-8.

23. Hallmann L, Mehl A, Sereno N, Hammerle C. The improvement of adhesive properties of PEEK through different pretreatments. Appl Surf Sci. 2012;258:72138.

24. Goyal RK, Tiwari AN, Negi YS. High performance polymer composites on poly ether ether ketone 
reinforced with Al2O3. J Appl Polym Sci.2006;100:4623-31.

25. Keul C, Liebermann A, Schmidlin PR, Roos M, Sener B, Stawarczyk B, et al. Influence of PEEK surface modification on the retention of two veneering resin composites. J Adhes Dent. 2013;32:441-8.

26. International organization for standardization. ISO standard 10477: dentistry-polymer-based crown and bridge materials Amendment;1996.

27. Mirza R, Baig A, Gunaseelan R, Norsiah Y. Zygomatic implant-retained fixed complete denture for an elderly patient. Gerodontology J. 2012;29:1140-5.

28. Stawarczyk B, Keul C, Beuer F, Roos M, Schmidlin PR. Tensile bond strength of veneering resins to PEEK: Impact of different adhesives. Dent Mater J. 2013; 32:441-8.

29. Jin-Soo A, Young A, Yoon L, Deog-G. Shear bond strength of MDP-containing self-adhesive resin cement and Y-TZP ceramics: effect of phosphate monomercontaining primers. BioMed Research Int. 2015;389234:6-12.

30. Peter T, Pontsa A. Attachment bonding technique using DTK adhesive. The Dent-Liner Summer. 2010;14:2505.

31. Patrick R, Schmidlin PR, Bogna St, Marco W, Thomas A, Christoph HF, et al. Effect of different surface pretreatments and luting materials on shear bond strength to PEEK. Dent mater. 2010;26:553-9.

32. Martin R, Verena P, Michael B, Nuno S, Carola K. Shear bond strength between veneering composite and PEEK after different surface modifications. Clin Oral Invest. 2015;19:739-44.

33. Rosentritt M, Behr M, Thaller C, Rudolph H, Feilzer A. Fracture performance of computer-aided manufactured zirconia and alloy crowns. Quintessence Int. 2009; 40:655-62.

34. Albert FE, El-Mowafy OM. Marginal adaptation and microleakage of Procera All Ceram crowns with four cements. Int J Prosthet. 2004;17:529-35.

35. Camila S, Manthan P, Eric S. In vitro shear bond strength of three self-adhesive resin cements and a resin-modified glass ionomer cement to various prosthodontic substrates. Operat Dent. 2013;38:186-96.

36. Capa N, Ozkurt Z, Canpolat C, Kazazoglu E. Shear bond strength of luting agents to fixed prosthodontic restorative core materials. Aust D J. 2009; 54:334-40.

37. International Organization for Standardization. Dental materials - Testing of adhesion to tooth structure, ISO/TS11405; 2003.

38. Uhrenbacher J, Schmidlin PR, Keul C, Eichberger M, Roos M, Gernet W, et al. The effect of surface modification on the retention strength of polyetheretherketone crowns adhesively bonded to dentin abutments. J Prosthet Dent .2014; 6:1489-97.

39. Vrochari AD, Eliades G, Hellwig E, Wrbas KT. Curing efficiency of four self-etching, self-adhesive resin cements. Dent Mater. 2009;25:1104-8.

40. Patrick R, Schmidlin PR, Christine K, Malgorzata R, Beatrice S, Bogna ST, et al. Influence of PEEK surface modification on surface properties and bond strength to veneering resin composites. J Adhes Dent. 2014;16:383-92. 